\title{
Review of: "Early alpha/beta oscillations reflect the formation of face-related expectations in the brain"
}

\author{
Fabiano Baroni
}

Potential competing interests: The author(s) declared that no potential competing interests exist.

Roehe and colleagues reported EEG signatures related to implicit expectations in a face perception paradigm.

In line with previous findings, expectations were related to neural activity in the alpha/beta band, which was found to be related to subsequent neural processing of predicted vs. random faces as reflected in the N170 ERP and the gamma - high-gamma response.

The article constitutes an interesting contribution to a fast-growing literature of the relationships between pseudo-rhythmic brain activity and sensory predictions. Some of its features make it relevant with respect to several open questions of great interest in the object perception community, and more generally in cognitive neuroscience:

1) When discussing prediction mechanisms, a critical distinction is often drawn between predicting "when" versus "what" or, in other words, between temporal and spatial predictions (e.g., [1]). In this study, the authors made the relatively unusual choice of adopting a fixed Inter-Stimulus Interval: faces were presented at a fixed ISI of 3s, corresponding to a presentation frequency of $0.33 \mathrm{~Hz}$. Hence, the temporal aspect of stimulation was perfectly predictable.

On top of this perfectly rhythmic sequence of stimuli, face identity (the "what" component in their study) was manipulated so that a subset of face pairs were always presented contiguously, with the first (cueing) face invariably predicting the presentation of the second (predicted) face in the next trial for each pair. The comparison between the neural responses to predicted versus non-predicted (and non-cueing) faces constitutes the major part of the article. It remains an interesting topic for future work to assess whether their results are dependent on a rhythmic ISI, or whether they could also be observed in a more natural context, where the predictability of both "when" and "what" are manipulated independently.

2) The authors reported a complex spatio-temporal pattern of alpha-beta power increases when comparing the neural responses to predictive vs. non-predictive faces (Fig. 4), with peaks occurring shortly after the 
presentation of the predictive vs. non-predictive face, around the middle of the ISI, and near the end of the ISI, shortly before the presentation of the predicted vs. random face.

It would be interesting to know whether the temporal fluctuation in alpha-beta activity could be related to some slower rhythm, perhaps as a phase-amplitude cross-frequency coupling phenomenon with an endogenous slow rhythm or perhaps one entrained by the $0.33 \mathrm{~Hz}$ stimulation rate.

3) With respect to gamma band activity (GBA), the authors reported a decrease in gamma power in response to expected vs. random faces, which is especially conspicuous some $900 \mathrm{~ms}$ after stimulus onset (Fig. 3). The spectrogram shown in Fig. $3 \mathrm{~A}$ is hard to interpret though, since the greatest reduction is observed at or near the end of the time and frequency axes. It would have been useful to extend the time and frequency axes beyond their current limits, to show how the described phenomenon is limited in frequency and time (e.g., [2]).

As currently shown, it is possible that the GBA decrease is actually a broadband phenomenon. It is also not possible to discard a potential relationship with eye movements, which are known to increase high frequency activity [3]. There are no reasons to suppose that expected faces would result in more eye movements than random faces some $900 \mathrm{~ms}$ after stimulus onset, but it might still be worth doublechecking for this potential confound. Correction for eye movements is mentioned in the Methods, but it is not mentioned if there were differences in eye movements between conditions.

4) The time-frequency (TF) decomposition has been conducted in a manner which only preserves evoked (or time-locked) activity, since the authors state that "Spectral power was estimated by applying FFT to a sliding window passing through averaged trials". If averaging over trials is performed before applying the FFT, pseudo-oscillatory activity that is not time-locked to trial onset (often termed "induced" activity) is greatly reduced by averaging, with the result that only evoked activity is retained (e.g., [4]). This could perhaps have been emphasized more.

Assessing only evoked activity could be justified with respect to the GBA, given that the authors are especially interested in bottom-up activity which is likely to be time-locked; however, low frequency activity probably comprises a substantial induced (i.e., non time-locked) component.

5) The authors reported that subjects were first administered an 18-minute behavioral session (without concomitant EEG recording) to be exposed to the relevant sequential contingencies. Hence, the EEG data 
they showed reflects neural activity after subjects had already learnt these contingencies, at least to some extent. It remains an important topic for future work to characterize the time course of the behavioral and neurophysiological signatures underlying the implicit acquisition of these contingencies.

References

[1] Morillon, Benjamin, Charles E. Schroeder, Valentin Wyart, and Luc H. Arnal. "Temporal Prediction in Lieu of Periodic Stimulation." The Journal of Neuroscience : The Official Journal of the Society for Neuroscience 36, no. 8 (February 2016): 2342-47.

[2] Donoghue, Thomas, Natalie Schaworonkow, and Bradley Voytek. “Methodological Considerations for Studying Neural Oscillations." PsyArXiv, March 18, 2021. https://doi.org/10.31234/osf.io/hvd67.

[3] Yuval-Greenberg, Shlomit, Orr Tomer, Alon S. Keren, Israel Nelken, and Leon Y. Deouell. “Transient Induced Gamma-Band Response in EEG as a Manifestation of Miniature Saccades." Neuron 58, no. 3 (May 2008): 429-41. https://doi.org/10.1016/j.neuron.2008.03.027.

[4] Tallon-Baudry, C., O. Bertrand, F. Peronnet, and J. Pernier. "Induced Gamma-Band Activity during the Delay of a Visual Short-Term Memory Task in Humans." The Journal of Neuroscience: The Official Journal of the Society for Neuroscience 18, no. 11 (June 1998): 4244-54. 